\title{
Gestão de processos de negócio e configuração de valor para consultoria de alto desempenho
}

\begin{abstract}
Este artigo tem por objetivo apresentar a aplicação do conceito de oficina de valor, um tipo de configuração de valor, no âmbito da gestão de processos de negócios. Inicialmente, revisou-se a literatura sobre configuração de valor, gestão de processos de negócio e geração de valor em consultorias, depois entrevistas foram realizadas com especialistas e com as partes interessadas de uma organização que colaborou com a aplicação desta pesquisa. Em seguida, foi modelada a oficina de valor na organização pesquisada, uma consultoria empresarial. Por fim, foram desenhados e implementados os processos de negócio, em que foram avaliados os impactos no desempenho organizacional da consultoria. Para o mapeamento dos processos, optou-se pelo desenho da situação ideal sem o desenho da situação atual, devido ao pouco prazo que a empresa dispunha para a pesquisa, não foram verificados quaisquer prejuízos metodológicos quanto a esta escolha. O resultado é um modelo integrado de processos alinhado a estratégia organizacional que está em funcionamento há dez anos. O procedimento técnico Pesquisa em Ciência do Projeto, ou Design Science Research, alinhado ao procedimento pesquisa-ação garantiu a disseminação do conhecimento, sendo bem avaliado pelas partes interessadas e contribuindo para o desenvolvimento de um modelo de consultoria de alto desempenho.
\end{abstract}

Palavras-chave: Gestão de Processos de Negócio; Gestão de Projetos; Configuração de Valor; Gestão da Mudança.

\section{Business process management and value configuration for high performance consulting}

\begin{abstract}
This article aims to present the application of value office concept, a value configuration type within business process management. Initially, was reviewed literature in value configuration, business processes management and value creation in consulting, after interviews were conducted by experts and stakeholders of an organization that collaborated with the application of this research. Then value office was modeled in a business consulting. Finally, we designed and implemented business processes, which evaluated the impacts on organizational performance consulting. For the processes mapping, we opted for to be mapping without as is mapping, given the short time that this company had for the survey were not verified any methodological losses. The result is an integrated process model aligned with organizational strategy that has been operating for ten years. The technical procedure design science research, in line with the procedure action research ensured the knowledge dissemination, being well evaluated by stakeholders and contributing to the development of a high performance consulting model.
\end{abstract}

Keywords: Business Process Management; Project Management; Value Configuration; Change Management.

Topic: Operações e Processos da Produção

Reviewed anonymously in the process of blind peer.

Karoll Haussler Carneiro Ramos

Universidade de Brasília, Brasil

http://lattes.cnpq.br/5981443802221449

karoll.ramos@gmail.com

Sueli Menelau de Novais

Universidade de Brasília, Brasil

http://lattes.cnpq.br/8006331919726232

suelimenelau@gmail.com
Received: 10/06/2016

Approved: 23/11/2016
Referencing this:

RAMOS, K. H. C.; NOVAIS, S. M.. Gestão de processos de negócio e configuração de valor para consultoria de alto desempenho. Revista Brasileira de Administração Científica, v.7, n.3, p.170-186, 2016. DOI: http://doi.org/10.6008/SPC2179-684X.2016.002.0012 


\section{INTRODUÇÃO}

Para responder ao cenário atual de alta complexidade, os modelos organizacionais são desenvolvidos para aumentar a agilidade de resposta das organizações frente as mudanças dos cenários externos. Em uma época em que os cenários externos eram mais previsíveis do que os atuais, os modelos organizacionais, como os de Taylor e de Fayol, eram centrados em estruturas de trabalho e em funções organizacionais, contribuindo para uma visão verticalizada do negócio (RUMMLER; BRACHE, 1994). Tal visão que enfatiza estruturas hierárquicas e divisão por departamentos ou áreas, condiciona os executivos à gestão de 'silos' em que o desempenho organizacional é, simplesmente, o somatório do desempenho individual das unidades organizacionais (RUMMLER; BRACHE, 1994; MCCORMACK, 2007).

Nos dias atuais, as organizações buscam se orientar em modelos que tenham capacidade de interagir com sistemas cada vez mais complexos e instáveis - como ambientes socioeconômicos, políticos, tecnológicos, culturais entre outros -, que findam em descaracterizar as relações inter e intra organizacionais dos arquétipos mais comuns (CLEGG; HARDY, 2012). Dentre essas abordagens emergentes de gestão, a gestão de processos de negócio (do inglês Business Process Management - BPM) vem sendo amplamente utilizada como instrumento para identificar, avaliar e melhorar as organizações por meio da orientação dos Processos de Negócio (PN) aos resultados organizacionais e às necessidades mercadológicas, em oposição às hierarquias voltadas a si mesmas (MCCORMACK, 2007).

Durante a implementação do BPM nas organizações destaca-se o uso da cadeia de valor de Porter (1989) que, segundo o autor, é uma representação sistemática da interação das atividades executadas que envolvem capacitações e competências tecnológicas de relevância estratégica à organização e ao seu negócio. Um dos alicerces da vantagem competitiva, a BPM traz benefícios tangíveis e intangíveis às organizações por desagregar suas atividades e com isso estruturar os processos de uma organização encadeando-os em uma lógica de obtenção de valor (PORTER, 1989). Entretanto, cabe ressaltar que essa estrutura pode não representar a lógica da criação de valor de um negócio, uma vez que esta configuração de valor fora desenvolvida para linhas de produção para indústrias manufatureiras (STABELL; FJELDSTAD, 1998; GOTTSCHALK; AALST; JANSEN-VULLERS; LA ROSA, 2008; GOTTSCHALK; LA ROSA, 2010)

Uma das formas de se abordar o BPM é direcionar o foco da investigação à lógica de configuração de valor que melhor se adeque ao negócio, mas que também esteja alinhada as necessidades dos clientes e aos próprios resultados da organização. Nas pesquisas sobre BPM são poucos os estudos que analisam a lógica da criação de valor dos processos quanto ao uso da configuração de valor mais adequada a cada negócio. Destacam-se os estudos de Stabell e Fjeldstad (1998), Gottschalk et al. (2008) e Gottschalk e La Rosa (2010) que, contudo, não realizaram pesquisa aplicada, apenas pesquisa teórica. Vale ressaltar ainda que $60 \%$ a $80 \%$ das iniciativas em BPM são insucesso (KARIM; SOMERS; BHATTACHERJEE, 2007; ABDOLVAND; ALBADVI; FERDOWSI, 2008) e são altas as taxas de erros nas implementações (ARIYACHANDRA; FROLICK, 2008; LEE; AHN, 2008). Logo, há muito potencial em incrementos à BPM que resultem em iniciativas bem-sucedidas. 
Este estudo tem por objetivo aplicar a análise de configuração de valor à abordagem BPM com vistas a desenvolver um modelo integrado de processos para a melhoria do desempenho organizacional. Para esta pesquisa empírica, utilizou-se a metodologia Design Science em uma consultoria empresarial. Utilizou-se as fases de BPM apresentadas por de Segatto, De Pádua e Martinelli (2013). O artigo está organizado nas seguintes seções, além desta introdutória: a Seção 2 fornece a revisão da literatura, sendo dividida em três subseções que apresentam a Gestão de Processos de Negócio, a Configuração de Valor e a Análise de Valor em Consultoria; a Seção 3 discute a metodologia; na Seção 4 são apresentados os resultados da pesquisa que na Seção 5 são discutidos. Por fim, na Seção 6 estão sumarizados as descobertas e os resultados.

\section{REFERENCIAL TEÓRICO}

\section{Gestão de Processos de Negócio}

A teoria sobre gestão de processos de negócio ainda hoje é heterogênea e segmentada, não sendo possível definir claramente seus limites pois, segundo Antunes e Mourão (2011), o que existe são especulações sobre origem, objetivos, justificativas, benefícios e, até mesmo campo de pesquisa. Ainda de acordo com os autores, a discussão sobre a origem do BPM começa com a racionalização dos trabalhos de Taylor e de Gantt, tendo a revolução industrial propulsionado a inovação dos processos, sendo acelerada em 1990 com o largo uso dos sistemas integrados (do inglês Enterprise Resource Planning - ERP). Já para Schmiedel, Brocke e Recker (2014), o BPM surgiu em oposição à visão funcional originada da divisão do trabalho no Tailorismo. Entretanto, diferente de Antunes e Mourão (2011), alguns autores consideram a década de 1990 como o início do BPM (BUHL; RÖGLINGER; STÖCKL; BRAUNWARTH, 2011; ROESER; KERN, 2014). A consideração por tal período está fundamentada nos trabalhos de Davenport e Short (1990) e de Hammer e Champy (1993), que enfatizam a melhoria dos processos de negócio.

Sobre os objetivos do BPM, existe a visão de aceleração dos processos organizacionais, redução das necessidades de recursos, melhoria da competitividade das organizações (BAl; SARKIS, 2013), melhoria da eficiência e da eficácia dos processos organizacionais por meio de melhorias ou inovações (SCHMIEDEL; VOM BROCKE; RECKER, 2014), integração entre atividades e tecnologia (ANTUNES; MOURÃO, 2011), técnicas para sofisticação dos sistemas de planejamento, de monitoramento e de controle que se alinham a estratégia de gestão da qualidade (SLAVICEK, 2011). Também pode-se observar na literatura o estreito relacionamento entre BPM e gestão da qualidade total (do inglês Total Quality Management - TQM).

Para Roeser e Kern (2014) essa aproximação entre os temas influencia na abertura de dois tipos de abordagem em BPM, sendo uma voltada para o ciclo PDCA (Plan, Do Check e Act) e outra direcionada à abordagem para gestão. Esta pesquisa considera a abordagem voltada ao ciclo PDCA e que enfatiza o ciclo de BPM composto de seis fases que, conforme Segatto, De Pádua e Martinelli (2013), possuem os seguintes significados: (a) No Planejamento são desenvolvidos planos e estratégias que visam processos organizacionais. Nesta fase, são compreendidos estratégias e objetivos da organização, fornecendo orientações à gestão dirigida aos clientes e ao alinhamento com a estratégia organizacional, bem como a 
integração de pessoal, de processos, de sistemas e de sistemas de desempenho; (b) Na Análise são compreendidos os processos organizacionais em voga, são assimiladas as informações a partir planejamento estratégico, e são definidos os modelos de processos, a avaliação de desempenho e as mudanças no ambiente organizacional; (c) Na Modelagem envolve-se a criação de representações existentes ou propostas de processos de negócios por meio da documentação da sequência de atividades para fornecer valor ao cliente. Nesta fase, o desenvolvimento do processo está documentado, incluindo melhorias num ciclo de vida de BPM iterativa. As atividades iniciais do projeto podem se concentrar em padronização ou automatização das atividades ad hoc, ou melhorias incrementais projetada para otimização; (d) Na Implementação são postas em prática as atividades definidas pelos processos; (e) No Monitoramento e Controle são fornecidas informações aos gestores para que possam ajustar os recursos de acordo com os objetivos, gerando assim informação sobre o desempenho por meio de métricas relacionadas aos objetivos da organização; (f) No Refinamento são realizadas atividades de melhoria, incluindo remodelagem resultante da análise das informações sobre o desempenho do processo.

A aplicação do ciclo de fases de BPM entende que os processos devem ser continuamente melhorados (SEGATTO; DE PÁDUA; MARTINELLI, 2013). Assim, uma vez finalizado o primeiro ciclo de BPM deve ser reiniciada uma nova fase de Planejamento e consecutivamente, galgando assim maturidade da gestão de processos.

\section{Configurações de Valor}

Configurações de valor são representações que mostram a lógica da criação de valor das organizações, representada por meio das atividades de valor da organização ou macroprocessos (STABELL; FDELDSTAD, 1998). São três as configurações de valor: cadeia de valor, oficina de valor e rede de valor. Stabell e Fdeldstad (1998) associam as configurações de valor com as tecnologias de Thompson (1967). Para Thompson (1967) tecnologia é o conjunto ordenado de conhecimentos empregados na produção e comercialização de bens e serviços que integra conhecimentos científicos e empíricos, que resultam de observações, experiências, atitudes específicas, tradição (oral ou escrita) (RODRIGUES, 1984).

Thompson (1967 citado por STABELL; FDELDSTAD, 1998), define três tipos de tecnologias nas organizações: elos em sequência, mediação e intensiva. Essas tecnologias são categorizadas conforme a independência de tarefa - baixa, média e alta -, sendo que cada uma é fortemente associada a um tipo de configuração de valor. Assim, o resultado de tal associação considera: (i) cadeia de valor correspondente à tecnologia elos em sequência; (ii) oficina de valor à tecnologia intensiva; e (iii) rede de valor vinculada à tecnologia de mediação. Para este estudo duas configurações de valor a serem estudadas são cadeia de valor e oficina de valor. Ambas as configurações são organizadas em atividades primárias e atividades de apoio.

As atividades primárias ou de negócio representam as atividades necessárias à produção de um produto ou de um serviço final que, conforme Piemonte (2011), podem ser identificadas por meio da correlação com a missão da organização, com a percepção da qualidade oferecida ao cliente e pelo sequenciamento temporal das atividades. Além disso, as configurações de valor se diferenciam pelo 
conteúdo e sequenciamento dessas atividades primárias. Já as atividades de apoio fornecem insumos para a realização das atividades primárias, tendendo a ser as mesmas para as configurações de valor, como por exemplo, infraestrutura, gerência de recursos humanos, desenvolvimento de tecnologia e aquisição (PORTER, 1998). Ressalta-se que esta pesquisa se limita ao desenvolvimento das atividades de negócio, justamente por se considerar o seu impacto no contexto organizacional.

Segundo Porter (1998), a cadeia de valor possui atividades de valor e margem, sendo a margem a diferença entre valor total e o custo coletivo da execução das atividades de valor, e seu isolamento entendido como importante para a compreensão das fontes da posição de custo de uma empresa (Figura 1).

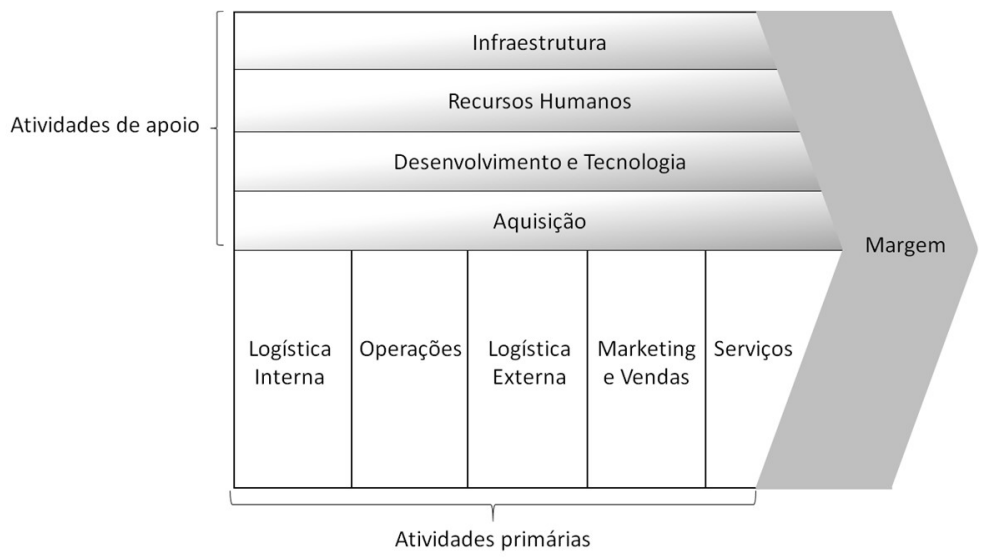

Figura 1: Cadeia de Valor. Fonte: Porter (1998, p.30).

A Figura 1 mostra cinco atividades primárias da cadeia de valor: logística interna; operações; logística externa; marketing e vendas; e serviços (PORTER, 1989). Já a configuração de valor do tipo oficina de valor (Figura 2), proposta por Stabell e Fjeldstad (1998), confia em uma tecnologia intensiva para resolver os problemas do cliente.

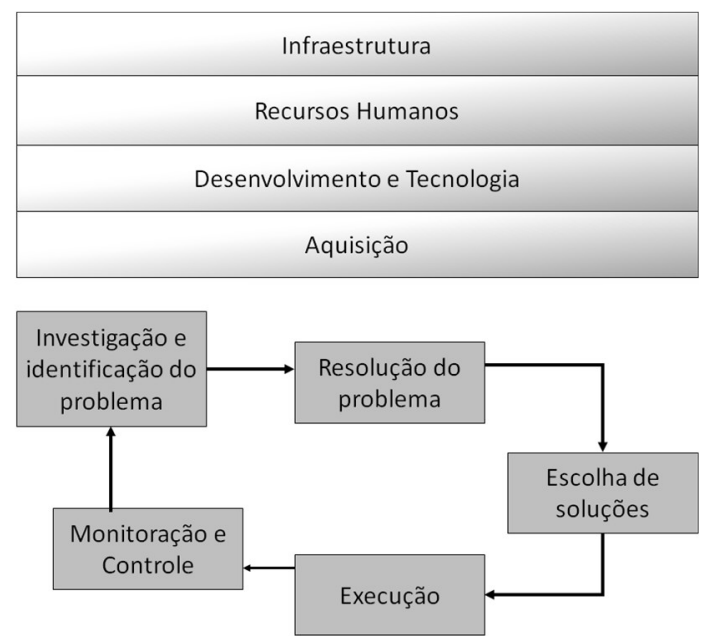

Figura 2: Oficina de Valor.

Fonte: Adaptado de Stabell e Fjeldstad (1998, p.424).

A Figura 2 mostra cinco as atividades primárias da oficina de valor: (i) definição de problema, que diferencia as causas que geram o problema percebido; (ii) resolução do problema, que identifica as atividades associadas com a gravação e a formulação do problema a ser resolvido; (iii) escolha de soluções, 
que gera, avalia e escolhe soluções alternativas para o problema; (iv) execução da solução, que realiza e comunica a organização; e (v) controle e a avaliação, que verificam o impacto das soluções implementadas na organização, por meio da medição do desempenho e da avaliação da solução proposta (STABELL; FJELDSTAD, 1998).

A seleção, a combinação e a ordem da aplicação dos recursos e das atividades variam de acordo com as exigências do problema. Assim, enquanto a cadeia de valor se concentra em um conjunto de atividades para produzir um produto padronizado em grande escala, a oficina de valor programa as atividades e a aplicação dos recursos sobre as necessidades do problema do cliente (STABELL; FJELDSTAD, 1998; GOTTSCHALK et al., 2008; GOTTSCHALK; LA ROSA, 2010).

\section{Análise da Lógica de Valor em Consultoria}

De acordo com Block (2000), existem cinco fases preliminares de um projeto de consultoria: (i) entrada e contrato; (ii) descobertas e diálogo; (iii) feedback e decisão para execução; (iv) engajamento e implementação; e (v) extensão ou término. A entrada e contrato inicia-se com o primeiro contato entre cliente e consultoria, na qual são explorados os problemas do cliente, quem serão os consultores com perfil para o projeto, levantamento de quais são as expectativas do cliente e dos consultores e como o projeto deverá iniciar. Na descoberta e diálogo os consultores obtêm seu próprio senso do problema e ajudam seus clientes a fazerem o mesmo.

No feedback e decisão para execução, o levantamento de dados e a análise devem ser relatados. Nessa fase há também escolhas para o consultor em como envolver o cliente no processo de análise da informação. $O$ engajamento e implementação abarca o envolvimento e o planejamento da próxima etapa. Alguns projetos começam com a participação do cliente em algum evento educacional para introduzir algumas mudanças, mas pode-se requerer uma única reunião de partes diferentes da organização e assim descobrir um problema. Destaca-se que para grandes mudanças, os consultores deverão estar envolvidos. A extensão ou término começa com a avaliação da fase anterior e seguirá com a decisão de estender o processo para mais segmentos da organização.

Por sua vez, Rummler (2004) determina que o projeto de consultoria envolva quatro fases, cada uma possuindo objetivos e produtos específicos, quais sejam: (i) resultados determinados e projeto definido; (ii) barreiras determinadas e mudanças especificadas; (iii) mudanças planejadas, desenvolvidas e implementadas; e (iv) resultados avaliados, mantidos ou melhorados. Ainda para esse autor, cada projeto deve cumprir parcialmente ou completamente essas quatro fases, explicitadas no Quadro 1 a seguir.

Quadro 1: Fases do Projeto de Consultoria.

\begin{tabular}{|c|c|c|c|c|}
\hline & $\begin{array}{c}\text { Fase I } \\
\text { Resultados } \\
\text { determinados e o } \\
\text { projeto definido }\end{array}$ & $\begin{array}{c}\text { Farreiras determinadas e } \\
\text { mudanças especificadas }\end{array}$ & $\begin{array}{c}\text { Fase III } \\
\text { Mudanças planejadas, } \\
\text { desenvolvidas e } \\
\text { implementadas }\end{array}$ & $\begin{array}{c}\text { Fase IV } \\
\text { Resultados avaliados } \\
\text { mantido ou melhorados }\end{array}$ \\
\hline Objetivos & $\begin{array}{c}\text { Determinar se há } \\
\text { problemas } \\
\text { significativos a serem } \\
\text { resolvidos }\end{array}$ & $\begin{array}{c}\text { Identificar os fatores que } \\
\text { causam os problemas }\end{array}$ & $\begin{array}{c}\text { desenvolvimento e } \\
\text { implementações das }\end{array}$ & $\begin{array}{c}\text { Determinar se os problemas } \\
\text { foram resolvidos, caso não } \\
\text { quais os procedimentos } \\
\text { para solucioná-los }\end{array}$ \\
\hline
\end{tabular}




\begin{tabular}{|c|c|c|c|c|}
\hline \multirow{1}{*}{$\begin{array}{c}\text { Determinar a } \\
\text { possibilidade em } \\
\text { resolver os } \\
\text { problemas }\end{array}$} & $\begin{array}{c}\text { Especificar as mudanças } \\
\text { para resolver os } \\
\text { problemas } \\
\begin{array}{c}\text { Preparar um plano } \\
\text { de projeto que } \\
\text { resolverá os } \\
\text { problemas }\end{array}\end{array}$ & $\begin{array}{c}\text { intervenções necessárias e } \\
\text { assegurar melhoria contínua }\end{array}$ & \\
\hline Produtos & $\begin{array}{c}\text { Plano do projeto } \\
\text { Aceite do }\end{array}$ & $\begin{array}{c}\text { Recomendações para a } \\
\text { mudança }\end{array}$ & & Resultados levantados \\
& Procedimento & $\begin{array}{c}\text { Macro Design ou } \\
\text { Estrutura Analítica do } \\
\text { Projeto (EAP), Plano de } \\
\text { Implementação }\end{array}$ & Implementação das mudanças & \\
\hline
\end{tabular}

Fonte: Elaborado a partir de Rummler (2004).

Reunindo as propostas de Block (2000) e de Rummler (2004), foi elaborado um framework de seis fases que descreve a estruturação do trabalho de uma consultoria, onde também foram identificados os produtos (a lógica de valor) gerados em cada uma dessas fases (Quadro 2).

Quadro 2: Estrutura para Elaboração do Modelo de Consultoria.

\begin{tabular}{|c|c|c|c|c|c|c|}
\hline Contribuição & $\begin{array}{l}\text { Fase } 1 \\
\text { Contato } \\
\text { Inicial } \\
\end{array}$ & $\begin{array}{c}\text { Fase } 2 \\
\text { Diagnóstico }\end{array}$ & $\begin{array}{c}\text { Fase } 3 \text { Produto } \\
\text { Executado }\end{array}$ & $\begin{array}{c}\text { Fase } 4 \\
\text { Implementação }\end{array}$ & Fase 5 Avaliação & $\begin{array}{c}\text { Fase } 6 \\
\text { Controle e } \\
\text { Melhoria } \\
\end{array}$ \\
\hline Artefato 1 & $\begin{array}{l}\text { Entrada e } \\
\text { contrato }\end{array}$ & $\begin{array}{l}\text { Descobertas e } \\
\text { diálogo } \\
\text { feedback e } \\
\text { decisão para } \\
\text { execução }\end{array}$ & Não consta & $\begin{array}{l}\text { Engajamento e } \\
\text { implementação }\end{array}$ & Extensão ou término & Não consta \\
\hline \multirow{2}{*}{ Artefato 2} & \multirow{2}{*}{ Não consta } & $\begin{array}{c}\text { Resultados } \\
\text { determinados e } \\
\text { o projeto } \\
\text { definido }\end{array}$ & \multirow{2}{*}{$\begin{array}{l}\text { Mudanças } \\
\text { planejadas, } \\
\text { desenvolvidas }\end{array}$} & \multirow{2}{*}{$\begin{array}{c}\text { Mudanças } \\
\text { implementadas }\end{array}$} & \multirow{2}{*}{ Não consta } & \multirow{2}{*}{$\begin{array}{l}\text { Resultados } \\
\text { avaliados } \\
\text { mantido ou } \\
\text { melhorados }\end{array}$} \\
\hline & & $\begin{array}{c}\text { Barreiras } \\
\text { determinadas e } \\
\text { mudanças } \\
\text { especificadas }\end{array}$ & & & & \\
\hline \multirow{4}{*}{$\begin{array}{l}\text { Produto } \\
\text { gerado }\end{array}$} & \multirow{4}{*}{$\begin{array}{l}\text { Aceite para } \\
\text { diagnóstico }\end{array}$} & $\begin{array}{l}\text { Quadro dos } \\
\text { problemas da } \\
\text { organização }\end{array}$ & $\begin{array}{l}\text { Implantação da } \\
\text { gestão da } \\
\text { Mudança } \\
\end{array}$ & \multirow{4}{*}{$\begin{array}{l}\text { Resultado do } \\
\text { Protótipo }\end{array}$} & \multirow{4}{*}{$\begin{array}{l}\text { Avaliação do projeto } \\
\text { pelo cliente e } \\
\text { equipe, nesta fase } \\
\text { será avaliado se há } \\
\text { necessidade de mais } \\
\text { produtos e/ou de } \\
\text { um novo contrato }\end{array}$} & \multirow{2}{*}{$\begin{array}{l}\text { Quadro de } \\
\text { avaliação de } \\
\text { resolução dos } \\
\text { problemas da } \\
\text { empresa. }\end{array}$} \\
\hline & & Quadro da & \multirow{3}{*}{ Produto } & & & \\
\hline & & $\begin{array}{l}\text { perspectiva de } \\
\text { desempenho } \\
\text { para o projeto. }\end{array}$ & & & & \multirow{2}{*}{$\begin{array}{l}\text { Quadro de } \\
\text { avaliação do } \\
\text { valor do } \\
\text { produto para a } \\
\text { organização }\end{array}$} \\
\hline & & Plano do Projeto & & & & \\
\hline
\end{tabular}

Fonte: Desenvolvido pelos autores a partir de Block (2000) e Rummler (2004).

Em suma, este framework apresentado no Quadro 2 busca conciliar as evidências dos dois modelos de BPM para que tanto os resultados organizacionais quanto as necessidades mercadológicas possam contribuir para o desempenho organizacional. Com esta motivação, apresenta-se em sequência a metodologia do estudo. 


\section{METODOLOGIA}

A abordagem adotada pela pesquisa foi do tipo qualitativa, conforme Rodrigues (2007). O estudo foi elaborado observando-se os preceitos de Hair, Babin, Money e Samouel (2005) para a pragmática de pesquisa em Administração, caracterizando-o como se segue: empírico, objetivo, analítico, dirigido pela teoria, lógico, rigoroso e parcimonioso. Com base nesse escopo, o plano de pesquisa foi determinado como descritivo e exploratório, pois tal estudo teve por objetivo analisar a adição da configuração de valor ao método de gestão de processos de negócio para a construção de um modelo integrado de processos (HAIR et al., 2005).

A natureza da pesquisa é aplicada com fundamentação em pesquisa básica, em que as fontes de informações se baseiam em pesquisa bibliográfica e de campo (RODRIGUES, 2007). A pesquisa utilizou os procedimentos de Ciência do Projeto (do inglês Design Science Research - DSR). A DSR se dedica a recomendar como construir artefatos que possuam certas propriedades desejadas que, segundo Simon (1996), refletem processos, produtos, papeis etc., a fim de fornecer orientação de especificação aos investigadores sobre uma determinada abordagem, criando normas para construção e validação de, por exemplo, frameworks, construtos, sistemas e modelos. Assim, o objetivo fundamental da DSR é desenvolver conhecimento para a concepção e o desenvolvimento de artefatos (VAN AKEN, 2004).

Van Aken (2004) determina que os artefatos sejam generalizáveis em classe de problemas, que de acordo com Lacerda et al. (2013, p. 744), "permitem que os artefatos e, por consequência, suas soluções, não sejam apenas uma resposta pontual a certo problema em determinado contexto". Para esta pesquisa, o artefato final foi a construção de um modelo integrado de processos desenvolvido por meio dos artefatos configuração de valor e gestão de processos de negócio, pertencentes à classe de problemas - gestão do desempenho (Quadro 3).

Quadro 3: Classe de Problemas e Artefatos.

\begin{tabular}{|l|ll|}
\hline Classe de Problemas & \multicolumn{2}{|l|}{ Artefatos } \\
\hline $\begin{array}{l}\text { Gestão do } \\
\text { desempenho }\end{array}$ & $\bullet$ & Configuração de valor \\
\hline
\end{tabular}

Uma vez que o objetivo do estudo é a aplicação da análise de configuração de valor à abordagem BPM a fim de desenvolver um modelo integrado de processos para a melhoria do desempenho organizacional, tornou-se necessário identificar um ambiente apropiado para experimentação. Também era imperativo que as informações fossem de fácil acesso e que pudessem ser obtidas a qualquer momento. Além disso, a empresa deveria ter interesse em experimentar abordagens que impactam no desempenho organizacional, em possuir ambiente favorável à gestão de processos e em engajar um grupo de pessoas para as reuniões de trabalho. Os sujeitos participantes dessa pesquisa foram indivíduos de uma consultoria empresarial da cidade de Brasília, do Distrito Federal (DF). Essa empresa, presente no mercado há 15 anos, atua apenas no DF, conta com mais de 60 colaboradores entre consultores e gestores. A consultoria oferta serviços para pequenas e médias empresas nas áreas de: marketing; organização e métodos; gestão de 
pessoas e finanças. Tem como parceiros Sebrae e empresas de consultoria empresarial, objetivando a troca de experiências e participação em projetos.

A pesquisa bibliográfica centrou-se na identificação dos procedimentos em Consultoria. Para isso, os trabalhos pesquisados foram dos autores Block (2000), Rummler (2004), McNamara (2006) e Segatto, De Pádua e Martinelli (2013). O instrumento de coleta de dados primários empregado constituiu-se em um roteiro de entrevista semiestruturada. Além dos dados primários, também foram buscados dados secundários, como documentos relacionados à gestão de projetos da organização. Na análise de dados secundários a análise documental se ateve ao planejamento estratégico, à cadeia de valor e ao portfólio. Já as entrevistas foram realizadas com três pesquisadores/professores da Universidade de Brasília, quatro parceiros, sete clientes e oito colaboradores da Consultoria.

O Projeto de Pesquisa, conforme a DSR, considerou os processos de construção de Peffers, Tuunanen, Rothenberger e Chartterje (2008) nos procedimentos adotados, quais sejam: Identificação do Problema, Definição dos Resultados Esperados, Projeto e Desenvolvimento, Demonstração, Avaliação e Comunicação. Esses processos adicionam às etapas do BPM elevados padrões de rigor com um alto nível de relevância, consequentemente, o projeto se torna relevante, mas também rigoroso em termos científicos (BLOCK, 2000; RUMMLER, 2004). Na Figura 3 estão mostrados os passos e especificados os procedimentos e instrumentos utilizados.

PROJETO DE PESQUISA

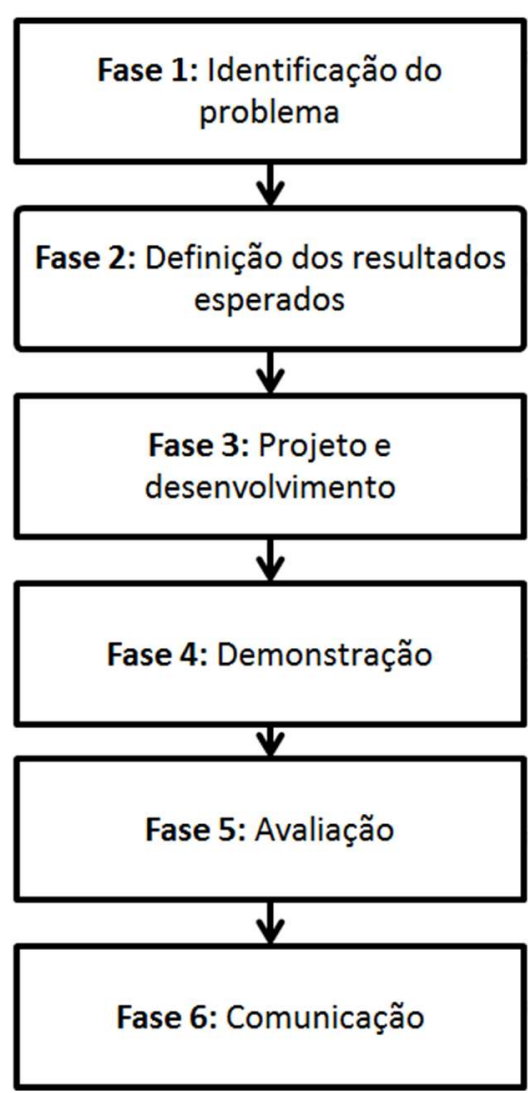

\section{PROCEDIMENTOS ASSOCIADOS}

1.Pesquisa documental

2. Entrevistas com clientes e partes interessadas

3. Pesquisa bibliográfica

1. Projeto básico de construção do Modelo de Consultoria alinhado à estratégia organizacional visando o alto desempenho

1. Elaboração da configuração de valor

2. Desenvolvimento das etapas do BPM

1. Desenvolvimento das etapas do BPM

1. Finalização das etapas do BPM

1. Apresentação e divulgação dos resultados

Figura 3: Fases do Projeto de Pesquisa da Ciência do Projeto. Fonte: Elaborado a partir de Peffers et al. (2008). 
Para a análise dos dados da pesquisa documental, das entrevistas e da pesquisa bibliográfica utilizouse análise de conteúdo categorial temática. A análise de conteúdo categorial temática se organiza em três fases: pré-análie; exploração do material e tratamento dos dados, inferência e interpretação (BARDIN, 2012). Já para a verificação da aplicação da gestão de processos de negócio foi cumprida as etapas do Projeto de Pesquisa da Ciência do Projeto, conforme a Figura 3.

\section{RESULTADOS}

\section{Fase 1: Identificação do problema}

As informações obtidas nessa primeira fase da pesquisa foram reunidas em dois grupos: 'oportunidades de melhoria' e 'o que deveria fazer e não estava fazendo'. No grupo 'oportunidades de melhoria' as informaç̧̃̃es obtidas foram categorizadas em seis grupos: (i) serviços ineficientes; (ii) erros na estimativa de tempo de projeto; (iii) alteração de escopo; (iv) erros de produtividade; (v) dificuldade na análise do valor gerado pelos produtos ao cliente; (vi) dificuldade na avaliação do serviço prestado.

A pesquisa documental mostrou que durante os 15 anos de funcionamento da Consultoria, a taxa de clientes que retornavam para fechar novos projetos era nula. Para o grupo de clientes entrevistados, o principal motivo para a não recontratação era a baixa resolução de seus problemas. Além disso, mencionouse a dificuldade na retenção de conhecimento e a dificuldade na implementação dos produtos entregues.

Dentre o grupo de parceiros e colaboradores, foram citadas falhas no processo ponta-a-ponta, ou seja, a cadeia de valor não era flexível apesar das constantes alterações de escopo. A organização, apesar de não ter os processos modelados, possuía rotinas bem definidas de gestão de projetos e da metodologia de seus serviços. Conforme os entrevistados, os serviços de consultoria se assemelhavam a uma fábrica de carros, em que o cliente reporta um problema, que não é investigado, e lhe são oferecidos os pacotes de serviços fechados. Assim, durante a realização dos projetos, os próprios consultores verificavam que o planejamento do projeto não atendia as necessidades dos clientes.

Ja no grupo 'o que deveria fazer e não estava fazendo' as informações coletadas, foram agrupadas em três categorias: Diagnóstico; Medição do Desempenho; e Retorno do Investimento dos Clientes.

Sobre o diagnóstico, os entrevistados relataram que a principal etapa de uma consultoria é a identificação das causas do problema. Para os entrevistados, os problemas não acontecem por si só, sendo o resultado de um conjunto de fatores ou variáveis falhas no sistema. Além disso, reforçaram que o diagnóstico contempla determinadas etapas de investigação, tais como: realizar o levantamento de informações, identificação ou constatação de causas, estudo de alternativas viáveis, proposição de soluções e, em alguns casos o acompanhamento e assessoramento da implementação das soluções sugeridas.

Por sua vez, a medição do desempenho é destacada pelos entrevistados como a etapa que deve ser planejada no processo de negociação. Nessa fase é combinado o que será medido para se avaliar o desempenho do serviço prestado. Para os entrevistados, no que se refere ao retorno do investimento dos clientes, houve ênfase na resposta dos entrevistados que citaram que a consultoria deve focar no retorno do 
investimento do cliente. Também foi mencionado pelos entrevistados o caso das consultorias boutique, que são consultorias de alto desempenho, uma vez que os valores negociados com o cliente estão atrelados ao retorno do investimento.

\section{Fase 2: Definição dos Resultados Esperados}

No período da realização desta pesquisa, a estratégia da consultoria empresarial era focada no desenvolvimento de soluções empresariais de excelência e transmissão de conhecimento, gerando valor para os clientes, parceiros e demais partes interessadas.

A ferramenta de planejamento estratégico que a consultoria utilizou é o balanced scorecard (BSC) que descreve as declarações estratégicas de uma organização, por meio de objetivos estratégicos definidos e dispostos em perspectivas distintas (KAPLAN; NORTON, 2001). A autorização para a realização desta pesquisa foi justificada na empresa por dois objetivos estratégicos: 'Aprimorar Serviços' e 'Garantir Qualidade dos Projetos'.

O BSC balanceia a utilização dos ativos intangíveis e tangíveis por meio de mapas de conexões de causa e efeito, denominados mapas estratégicos que norteiam o planejamento de níveis estratégico, tático e operacional (KAPLAN; NORTON, 2001). Conforme observado nesta empresa, na medida em que o planejamento estratégico é decomposto nos planos táticos e operacionais, os mesmos são implementados na organização, sendo readaptados conforme o balanceamento dos ativos e, consequente, provocando a readequação bottom-up da estratégia.

Assim, o projeto básico para esta pesquisa, que se refere, especificamente, ao plano tático e operacional do objetivo 'Aprimorar Serviços', propunha a construção de um modelo com vistas a suprir a percepção de uma cadeia de valor que não é flexível à execução dos serviços. Nesse sentido, era necessário verificar modelos que suprissem parcialmente as lacunas percebidas pelos resultados da fase anterior. Para isso, usou-se o Quadro 2 como fundamento para a identificação dos macroprocessos de negócio da Consultoria (Figura 4). A Figura 4 demonstra o desenho dos macroprocessos de negócio, alinhados a estratégia de negócio adotada pela organização estudada, segundo a lógica da oficina de valor.

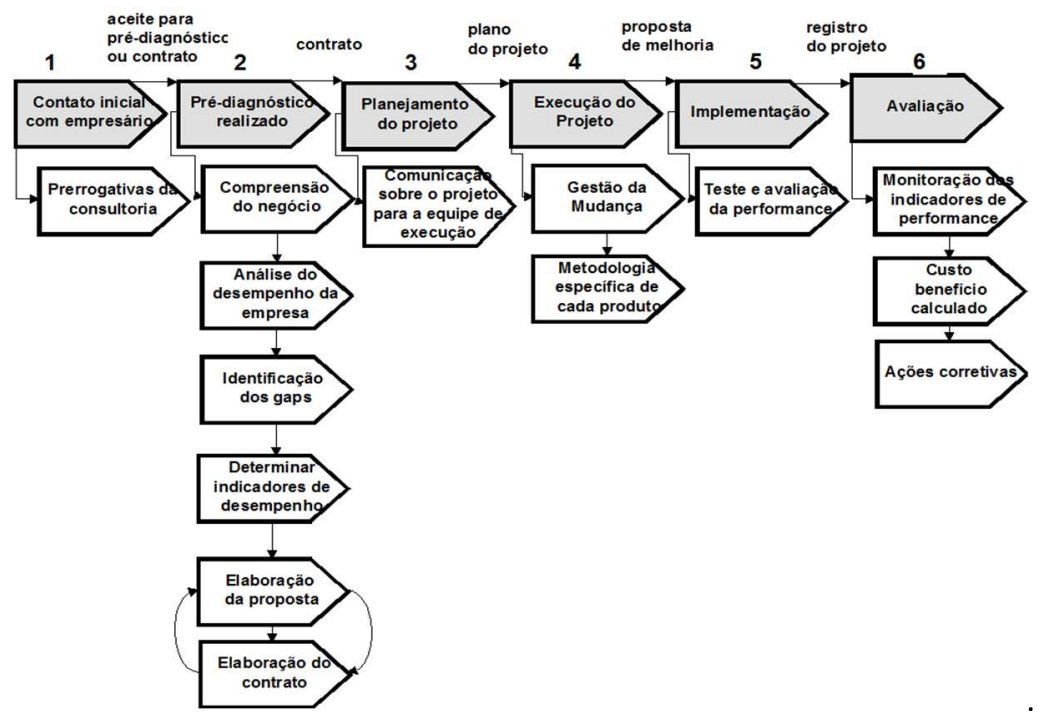

Figura 4: Macroprocessos de Consultoria 
Fase III: Projeto e Desenvolvimento

Para a fase de projeto e desenvolvimento, a Figura 4 foi rediscutida em workshops em que estavam presentes os membros da Diretoria Colegiada da empresa. Nesses encontros os macroprocessos foram reagrupados em três: macroprocesso Realizar Negócios que inclui os antigos macroprocessos Contato Inicial com Empresário e o Pré-Diagnóstico Realizado; macroprocesso Executar Projeto de Consultoria substituindo os macroprocessos Executar Projeto e Implementação e; o macroprocesso Avaliar Impacto do Projeto no Cliente no lugar do macroprocesso Avaliação. O novo rearranjo da oficina de valor é mostrado na Figura 5.

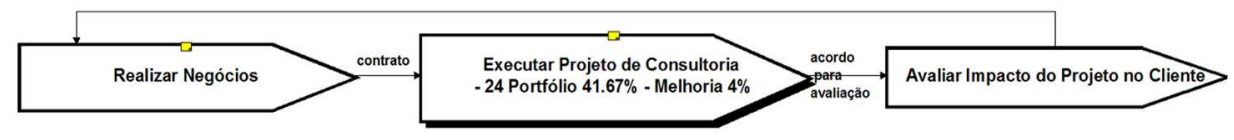

Figura 5: Macroprocessos da Oficina de Valor

Na Figura 5 estão descritos os macroprocessos de mais alto nível ou primeiro nível da oficina de valor. Depois, elaborou-se o segundo nível detalhando a primeira linha e, por fim a terceira linha que é a decomposição do segundo nível (Figura 5).

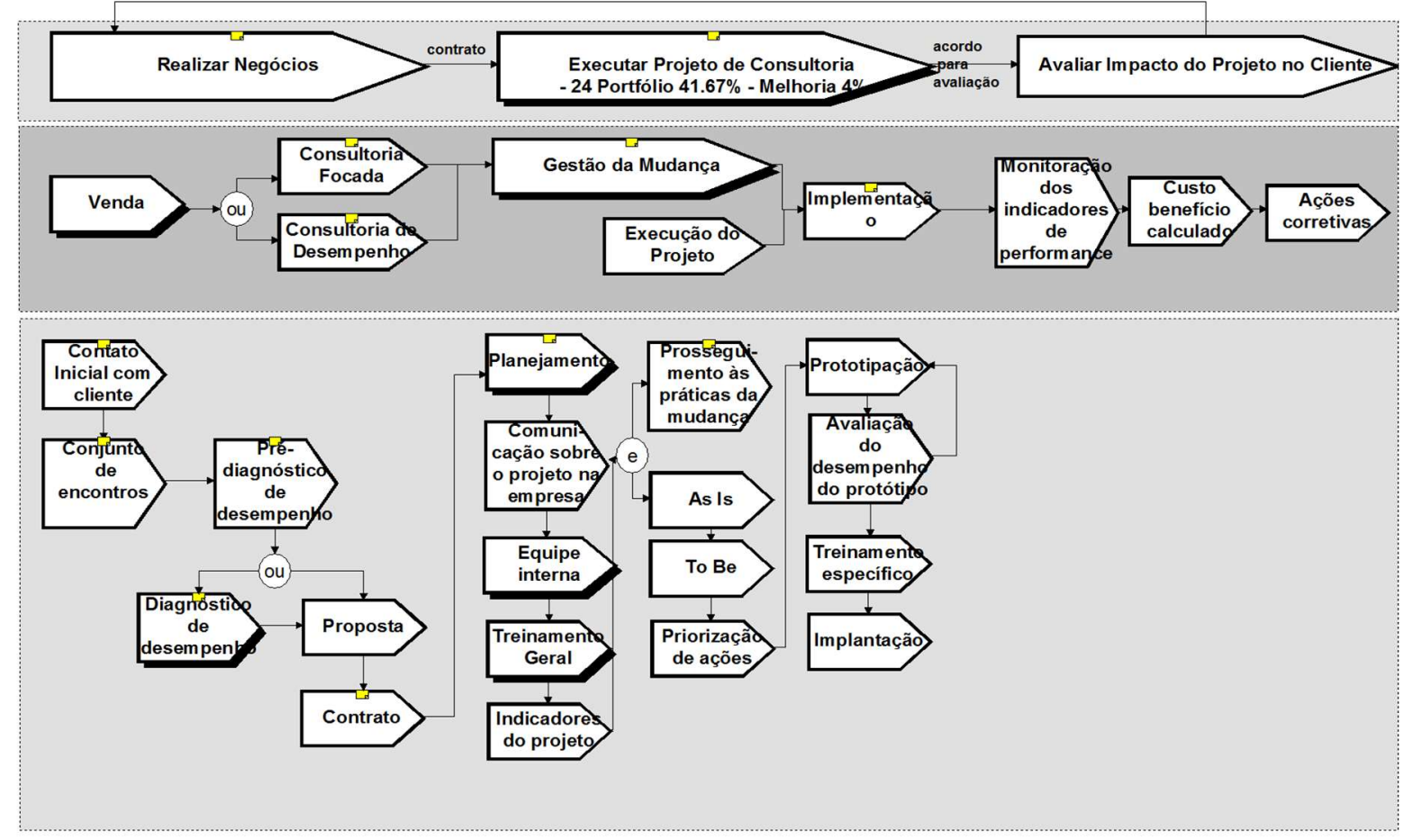

Figura 6: Oficina de Valor do Modelo de Consultoria.

O funcionamento desta representação é caracterizado pela sua flexibilidade, ou seja, poderão ocorrer casos em que não será necessária a tramitação por todo o processo. Esses casos existem devido a características do serviço ou pela forma de negociação.

O macroprocesso Realizar Negócios tem como valor principal o diagnóstico empresarial e para resolver a questão de adequação do diagnóstico para cada tipo de cliente, esse foi segmentado em dois: Consultoria Focada e Consultoria de Desempenho. Na Consultoria Focada fazem parte clientes com objetivos bem específicos, cuja necessidade não implica na averiguação das hipóteses mencionadas pelos clientes. 
$\mathrm{Na}$ Consultoria de Desempenho, como são reveladas dúvidas em relação ao objetivo da Consultoria, seria realizado o diagnóstico. Como há clientes que não precisam de um diagnóstico detalhado para a avaliação de seu estado, o problema do cliente seria categorizado em três níveis, conforme a complexidade dos problemas apresentados. Assim, todos os clientes passam pelo Nível 1, onde são coletados e analisados os problemas da empresa, caso o sistema de causa e efeito da organização seja prontamente identificado, a elaboração do escopo poderá ser definido, caso contrário o cliente seguirá ao Nível 2. Neste nível haverá o levantamento mais apurado da situação por meio de análise documental e se mesmo assim não for reconhecido o problema, passará para o Nível 3, onde o negociador desenhará uma estrutura de diagnóstico para aplicação.

No macroprocesso de Executar Projeto de Consultoria, a gestão da mudança proposta foi aprovada pelo Colegiado da Empresa e funciona como o catalisador de impactos, principalmente aos que afetam as pessoas, sejam elas do alto ou baixo escalão. No modelo proposto à gestão da mudança permearia o Antes, o Durante, a Implementação, por meio da implementação gradual dos processos.

O Levantamento Atual implica nas atividades de levantamento de informações pontuais referentes ao conhecimento a ser desenvolvido. Nesta fase a equipe interna trabalha auxiliando a equipe da Organização na explicação do funcionamento das atividades. Como percebido com o diagnóstico essa etapa é caracterizada como uma fase "morta" pelos clientes uma vez que o trabalho se restringe às validações da situação presente, aqui os Quick Wins podem surtir em um grande ganho e aprendizado. Segundo a observação do cliente, esse momento é o mais relevante de todo o projeto.

Antes da inicialização do desenho da situação desejada, o benchmarking agrega fortemente às ideias da Organização. Essa definição da situação desejada deve ser traçada juntamente com a equipe interna, uma vez que esta executará o novo procedimento e com as partes interessadas, pois os novos procedimentos poderão impactar nas interfaces horizontais e verticais da organização. É de fundamental importância que o novo desenho esteja alinhado ao negócio da organização.

A fase da implementação das ações do macroprocesso de Executar Projeto de Consultoria ocorre segundo as necessidades da organização. Inicialmente, os consultores participam do protótipo do projeto onde poderão coletar informações para possíveis ajustes e treinamento da equipe interna na nova gestão. Em seguida, a implementação efetiva será conduzida pelo líder do projeto e da equipe interna. O desempenho da equipe interna foi registrado e constam os indicadores para a fase de Avaliação e Controle. Após essas fases o trabalho da Organização está concluído.

Por fim, o macroprocesso de Avaliar Impacto no Cliente é um processo de avaliação dos resultados obtidos pela empresa com o projeto, por meio da análise dos indicadores de desempenho e verificam as novas oportunidades de melhorias a serem implementadas.

Vale ressaltar que cada fase apresentada do modelo de gestão de projetos é flexível conforme as características do cliente, ficando sob a responsabilidade de cada consultor de negócios e líder de projetos a adequação da negociação e escopo do projeto. 


\section{Fase IV: Demonstração}

A demonstração consta como etapa de prototipação. Inicialmente, realizou-se a implementação do macroprocesso Realizar Negócios. Para isso, o processo de diagnóstico foi priorizado. O diagnóstico deveria seguir determinadas etapas, consideradas fundamentais como compreensão do negócio, análise do desempenho da empresa, constatação do problema e apresentação do escopo.

Para esta implementação, uma empresa de médio porte, do setor atacadista foi selecionada. O projeto durou aproximadamente um mês e contou com uma equipe de cinco pessoas. Tal diagnóstico foi realizado em quatro etapas: compreensão do negócio, análise do desempenho da empresa, identificação das lacunas e determinação dos indicadores de desempenho do projeto.

Na compreensão do negócio foi verificado como os objetivos do negócio se comportam em relação às tendências do setor e compreender como esses afetam as atividades do negócio. Também foi apurado o alinhamento estratégico entre diretores e funcionários chave, que foi obtido por meio de reuniões de levantamento da missão, visão, produtos, clientes e fornecedores da empresa.

$\mathrm{Na}$ análise do desempenho da empresa foram apurados os problemas relatados pelo presidente da empresa durante o contato inicial com a Organização. Essa apuração se deu por meio da análise do ambiente interno da organização, considerando as várias áreas do conhecimento administrativo. Esta avaliação informou as características do sistema organizacional, uma vez que foram reveladas as variáveis que influenciam na organização no desempenho da organização.

$\mathrm{Na}$ identificação dos gaps, foram estabelecidas as relações de causa e efeito entre as variáveis que resultam em problema. A constatação do problema será confrontada com a os problemas apresentados pelo cliente na fase do contato inicial.

$\mathrm{Na}$ determinação de indicadores de desempenho para o projeto foi verificado quais indicadores seriam mensurados para um futuro controle e manutenção de um projeto que poderia ser fechado.

Durante a elaboração do diagnóstico, práticas de gestão da mudança foram realizadas com o objetivo de minimizar o pouco valor percebido pelo cliente. Assim, foi empregada a prática dos Quick Wins, que consiste no levantamento de pequenos pontos falhos na organização, cuja identificação ocorre com a fluidez ao longo do diagnóstico e a solução é simples, rápida e não compromete os resultados do projeto. Para este projeto piloto foram identificados mais de quarenta Quick Wins.

\section{Fase V: Avaliação}

Por meio dos resultados obtidos, validou-se que um diagnóstico bem estruturado é fonte de agregação de valor ao negócio, porque a solução proposta propicia o ciclo de melhoria contínua do cliente. Essa comprovação foi constatada, por meio dessa empresa cliente que até fechamento desta pesquisa encontra-se na terceira edição de projetos com a Empresa. 


\section{Fase V: Apresentação}

Após a realização do diagnóstico foi realizada uma apresentação sobre os resultados. Na apresentação participaram os executivos da Empresa. Como foram apontadas falhas em relação a determinadas áreas, parte dos participantes ficaram um pouco consternados, gerando posteriormente uma desmotivação com o trabalho e a queda na produtividade de alguns empregados em um período curto de tempo.

\section{DISCUSSÕES}

Acredita-se que os objetivos no que se refere à proposta do desenho de oficina de valor foram atingidos. Muitos problemas percebidos pela Organização eram ocasionados pela configuração de valor adotada, cadeia de valor. Essa cadeia é útil para linha de produção, porém quando aplicada em organizações demonstra ser um empecilho para o aumento do desempenho do cliente e da própria Organização.

Assim, quando se optou pela adoção do Modelo de Consultoria baseada em oficina de valor e gestão de processos de negócio houve um aumento da qualidade dos projetos entregues e a diminuição dos custos do projeto, uma vez que esse passou a ser customizados conforme a necessidade do cliente. Tal fato pode ser acompanhado ao longo de dez anos. Verifica-se que o Modelo de Consultoria continua a ser a base para o desenvolvimento de projetos da Empresa Pesquisada com pequenas alterações de leiaute e de suporte tecnológico.

A resolução de problemas adotados pela oficina de valor passou a considerar a organização como um modelo sistêmico em que uma variável pode afetar todo o sistema. Verificou-se que o Modelo de Consultoria a explora continuamente formas para melhorar a aprendizagem organizacional e a incorporação permanente de conhecimento, contribuindo para a tomada de decisão.

Após a implementação desse Modelo na organização, o cliente do projeto piloto procedeu com cinco renovações de contrato. Sobre a renovação de contrato com outros clientes, após esta pesquisa, passou a ser informação estratégica e sigilosa.

\section{CONCLUSÕES}

Esta pesquisa apresentou a aplicação da análise de configuração de valor à gestão de processos de negócios para o desenvolvimento de um modelo integrado de processos. Esse modelo, denominado Modelo de Consultoria é baseado em oficina de valor, desenvolvido com foco em alinhamento dos processos de negócio com a estratégia organizacional, com vistas ao aumento do desempenho organizacional. O Modelo de Consultoria foi testado em um cliente. O resultado permitiu a identificação sistêmica dos problemas do cliente, contribuindo para a elaboração de projetos com foco no desempenho organizacional.

O tratamento de um problema pela oficina de valor pode ser iniciado pela definição do problema e terminado na conclusão dessa fase, mas também pode ser continuado. Essa iteratividade cíclica ou elíptica 
das atividades da oficina de valor resulta em um grau elevado de interdependência sequencial e recíproca entre as atividades, diminuindo o custo de novos projetos.

O desafio é estabelecer indicadores significativos para mensurar o valor em uma situação onde se avalia a potencialidade da solução definida, a fim de se dirigir os futuros problemas do cliente ou os problemas potenciais que requerem soluções. A Organização deixou de ser unicamente a metodologia do portfólio de serviços, passando a ser também o domínio sobre o sistema organizacional do cliente. O que garante a identificação proativa dos projetos necessários para a melhoria do desempenho do cliente.

\section{REFERÊNCIAS}

ABDOLVAND, N.; ALBADVI, A.; FERDOWSI, Z.. Assessing readiness for business process reengineering. Business Process Management Journal, v.14, n. 4, p. 497-511, 2008. DOI: http://dx.doi.org/10.1108/14637150810888046

ANTUNES, P.; MOURÃO, H.. Resilient business process management: framework and services. Expert Systems with Applications, v. 38, n. 2, p. 1241-1254, 2011. DOI: 10.1016/j.eswa.2010.05.017

ARIYACHANDRA, T. R.; FROLICK, M. N.. Critical success factors in business performance management striving for success. Information Systems Management, v. 25, n. 2, p. 113-120, 2008. DOI:

http://doi.org/10.1080/10580530801941504

BAI, C.; SARKIS, J.. A grey-based DEMATEL model for evaluating business process management critical success factors. International Journal of Production Economics, v. 146 , n. 1, p. 281-292, 2013. DOI:

http://doi.org/10.1016/j.ijpe.2013.07.011

BARDIN, L.. Análise de conteúdo. São Paulo: Edições 70, 2011.

BLOCK, P.. Flawless consulting: a guide to getting your expertise lised. San Diego: Pfeiffer, 2000.

BUHL, H. U., RÖGLINGER, M., STÖCKL, S.; BRAUNWARTH, K. S.. Value orientation in process management: research gap and contribution to economically well-founded decisions in process management. Business and Information Systems Engineering, v. 3, n. 3, p. 163-72, 2011. DOI: http://doi.org/10.1007/s12599-011-0157-5

CLEGG, S. R.; HARDY, C.. Introdução: organização e estudos organizacionais. In: CLEGG, S. R.; HARDY, C.; NORD, W. R. Handbook de estudos organizacionais: modelos de análise e novas questões em estudos organizacionais. v. 1. São Paulo: Atlas, 2012. p. 29-58.

DAVENPORT, T. H.; SHORT, J. E.. The new industrial engineering: information technology and business process redesign. Sloan Management Review, Massachusetts, v. 31, n. 4, p. 11-27, 1990.

GOTTSCHALK, F.; AALST, W. M. P.; JANSEN-VULLERS, M. H.; LA ROSA, M.. Configurable workflow models. International Journal of Cooperative Information Systems, v.17, n. 2, p. 138, 2008. DOI: http://doi.org/10.1142/S0218843008001798
GOTTSCHALK, F.; LA ROSA, M.. Process configuration in YAWL. In: HOFSTEDE, A. H. M.; AALST, W. M. P.; ADAMS, M.; RUSSELL, N.. Modern business process automation: YAWL and its Support Environment. London: Springer, 2010. p.313382.

HAIR, J. F.; BABIN, B.; MONEY, A. H.; SAMOUEL, P.. Fundamentos de métodos de pesquisa em Administração. Porto Alegre: Bookman, 2005.

HAMMER, M.; CHAMPY, J.. Reengineering the corporation: a manifesto for business revolution. New York: HarperBusiness, 1994.

KARIM, J.; SOMERS, T. M.; BHATTACHERJEE, A. .The impact of ERP implementation on business process outcomes: a factor based study. Journal of Management Information Systems, v. 24, n.1, p.101-134, 2007. DOI: http://doi.org/10.2753/MIS0742-1222240103

KAPLAN, R. S.; NORTON, D. P.. Transforming the balanced scorecard from performance measurement to strategic management: Part II. American Accounting Association, v.15, n.2, p.147-160, 2001. DOI: http://doi.org/10.2308/acch.2001.15.2.147

LACERDA, D.P.; DRESCH, A.; PROENÇA, A.; ANTUNES JUNIOR, J. A. V.. Design Science Research: Método de pesquisa para a engenharia de produção. Gestão \& Produção, v.20, n.4, p.741-76, 2013.

LEE, S.; AHN, H.. Assessment of process improvement from organizational change. Information \& Management, v. 45, n. 5, p. 270-280, 2008. DOI: http://doi.org/10.1016/j.im.2003.12.016

MCCORMACK, K.. Business process maturity: theory and application. Charleston, SC: BookSurge Publishing, 2007.

MCNAMARA, C.. Field guide to consulting and organizational development: a collaborative and systems approach to performance, change and learning. Minneapolis, Minn: Authenticity Consulting, LLC, 2006.

MENELAU, S.; SANTOS, P. M. F.; CASTRO, B. G. A.; NASCIMENTO, T. G.. Realizar pesquisa sem ação ou pesquisa-ação na área de administração? Uma reflexão metodológica. Revista de Administração, São Paulo, v. 50, n.1, p.40-55, 2015. 
PORTER, M.. Vantagem competitiva: criando e sustentando um desempenho superior. Rio de Janeiro: Campus, 1998.

PEFFERS, K.; TUUNANEN, T.; ROTHENBERGER, M. A.; CHATTERJE, S.. A design science research methodology for information systems research. Journal of Management Information Systems, v. 24, n. 3, p. 45-78, 2008. DOI: http://doi.org/10.2753/MIS0742-1222240302

PIEMONTE, L. A.. Gestão da inovação: como integrar a gestão convencional, orientada ao desempenho com os novos requisitos de inovação e foco no cliente. São Paulo: Portal, 2010.

RODRIGUES, I. P. F.. Tecnologia, organização e rentabilidade: um modelo para investigação empírica. Revista de Administração de Empresas, v.24, n.4. p.63-69, 1984. DOI: http://dx.doi.org/10.1590/S0034-75901984000400012

RODRIGUES, R. M. Pesquisa acadêmica: como facilitar o processo de preparação de suas etapas. São Paulo: Atlas, 2007.

ROESER; T.; KERN; E. M.. Surveys in business process management - a literature review. Business Process Management Journal, v. 21, n. 3, p. 692-718, 2015. DOI: http://dx.doi.org/10.1108/BPMJ-07-2014-0065
RUMMLER, G.. Serious performance consulting: according to Rummler. Silver Spring: International Society for Performance Improvement, 2004.

RUMMLER, G.; BRACHE, A. P.. Melhores desempenhos das empresas. São Paulo: Makron Books, 1994.

SCHMIEDEL, T.; BROCKE, J. V.; RECKER, J.. Which cultural values matter to business process management?: Results from a global Delphi study. Business Process Management Journal, v.19, n. 2, p. 292-317, 2013. DOI: http://dx.doi.org/10.1108/14637151311308321

SEGATTO, M; PÁDUA, S. I. D.; MARTINELLI, D. P.. Business process management: a systemic approach? Business Process Management Journal, v. 19, n. 4, p. 698-714, 2013.

SLAVICEK, V.. Enhancing business process management with knowledge. E+M Ekonomie a Management, v.14, n.1, p.123, 2011.

STABELL, C. B., FJELDSTAD, $\varnothing$. D.. Configuring value for competitive advantage: on chains, shops and networks. Strategic Management Journal, v.19, p. 413-437, 1998. DOI: http://doi.org/10.1002/(SICI)10970266(199805)19:5<413::AID-SMJ946>3.0.CO;2-C 